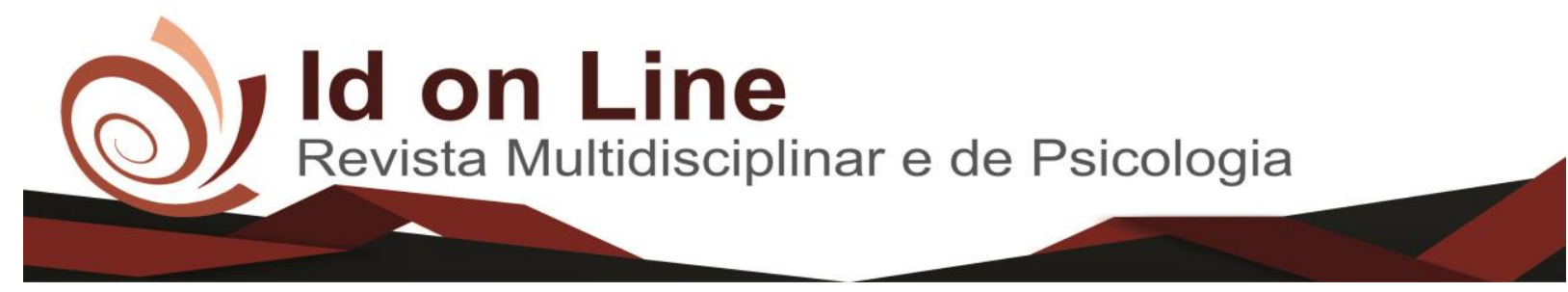

Artigo

\title{
Avaliar a Correlação da Obesidade com Hipertensão Arterial em Adultos Sedentários na Cidade de Vitória da Conquista
}

\author{
Larissa Santos Ormundo ${ }^{1}$; Stênio Fernando Pimentel Duarte ${ }^{2}$
}

\begin{abstract}
Resumo: O excesso de peso e a obesidade é um dos mais graves problemas de saúde pública dentro do grupo das doenças crônicas não transmissíveis contribuindo para o aparecimento de algumas comorbidades como as doenças cardiovasculares. O presente estudo teve como objetivo analisar a correlação da obesidade com a hipertensão arterial em adultos jovens. Trata-se de uma pesquisa do tipo de delineamento epidemiológico transversal, de cunho retrospectivo e descritivo e abordagem quantitativa. A população foi composta por 178 adultos jovens com idade entre 22 a 45 anos. Os participantes responderam um questionário sociodemográfico, a Escala de Silhuetas de Kakeshita, e por fim, foram submetidos a uma avaliação antropométrica. Houve associação significativa entre a obesidade e a hipertensão uma vez que de todos os pacientes avaliados os homens apresentaram cem por cento de hipertensão, enquanto as mulheres apresentaram um grau maior de faixa de risco de circunferência. Com isso, faz se necessárias investigações mais detalhadas através de exames físicos, laboratoriais e a medição da pressão arterial para melhores diagnósticos.
\end{abstract}

Palavras-chave: Obesidade, Circunferência abdominal, Hipertensão.

\section{To Evaluate the Correlation of Obesity with Arterial Hypertension in Sedentary Adults in the City of Vitória da Conquista}

\begin{abstract}
Overweight and obesity are one of the most serious public health problems within the group of chronic noncommunicable diseases contributing to the appearance of some comorbidities such as cardiovascular diseases. The present study aimed to analyze the correlation between obesity and hypertension in young adults. It is a research of the type of cross-sectional epidemiological design, with a retrospective and descriptive character and a quantitative approach. The population was composed of 178 young adults aged 22 to 45 years. Participants answered a sociodemographic questionnaire, the Kakeshita Silhouettes Scale, and finally, underwent an anthropometric evaluation. There was a significant association between obesity and hypertension once of all the evaluated patients, men presented one hundred percent hypertension, while women had a higher degree of circumference risk range. Therefore, more detailed investigations are necessary through physical, laboratory and blood pressure measurements for better diagnosis.
\end{abstract}

Key words: Obesity, Abdominal circumference, Hypertension

\footnotetext{
${ }^{1}$ Graduanda do Curso de Farmácia da Faculdade Independente do Nordeste - FAINOR. E-mail: laracle@ hotmail.com;

${ }^{2}$ Graduação em Ciências Biológicas na modalidade de Licenciatura Plena pela Universidade do Estado do Rio de Janeiro (2001), mestrado em Ciências (Fisiologia e Fisiopatologia Clínica e Experimental) pela Universidade do Estado do Rio de Janeiro (2002) e doutorado em Ciências (Fisiopatologia Clínica e Experimental) pela Universidade do Estado do Rio de Janeiro (2006). Tem experiência na área de Genética, com ênfase em Biologia Molecular e Fisiologia Humana, atuando principalmente com os temas: doenças crônicas, destacando a obesidade humana; polimorfismos genéticos e ataxias espinocerebelares. Atualmente atua como docente da Faculdade Independente do Nordeste - FAINOR e da Faculdade Tecnologia e Ciências - FTC.
} 


\section{Introdução}

A obesidade está cada vez mais frequente, sendo definida como uma doença crônica, que se caracteriza pelo excesso de gordura corporal e vem aumentando acentuadamente em países desenvolvidos e em desenvolvimento (Mariath et al., 2007). Tendo por causas conhecidas, um desequilíbrio nutricional provocado por uma maior ingestão calórica do que o organismo necessita, a forte influência do ambiente moderno, o comportamento sedentário, a diminuição dos níveis de atividade física, além de problemas genéticos, sendo por sua vez caracterizada como uma doença multifatorial. (Gharakhanlou, Farzad, Agha-Alinejad, Steffen, \& Bayati, 2012).

Segundo a Associação Brasileira para o Estudo da Obesidade e da Síndrome Metabólica (ABESO, 2017) a obesidade tem sido apontada como um grande problema de saúde pública para o mundo, com uma projeção de 2,3 bilhões de adultos com sobrepeso com mais 700 milhões de obesos para 2025. De acordo o Ministério da Saúde, no Brasil o número de pessoas com obesidade vem crescendo cada vez mais, no qual, levantamentos mostram que mais de $56,9 \%$ da população se encontra acima do peso, na região nordeste em especifico, o excesso de peso em adultos chega a $44,45 \%$.

Para que possa ser diagnosticado paciente obeso e consequentemente tratado ou, além disso, para que possa prevenir o sobrepeso ou a obesidade, é necessário realizar avaliações da condição de peso do paciente. Esse diagnostico se dá através das medidas antropométricas que pode ser pelo Índice de Massa Corporal (IMC), calculado através da divisão do peso em quilogramas $(\mathrm{kg})$ pela altura, em metros $(\mathrm{m})$, elevada ao quadrado, $\frac{P E S O}{A L T U R A^{2}}$; ou pela relação da circunferência da cintura (em metros) e do quadril (em metros) (RCQ), $\frac{\text { CINTURA }}{\text { QUADRIL }}$, quando associadas podem oferecer uma avaliação que ajude a diminuir as limitações de cada uma isoladamente (ABESO, 2016), (Souza et al., 2017).

A obesidade provoca diversas comorbidades associadas tais como doenças cardiovasculares (DCV), diabetes tipo 2, alguns tipos de câncer, bem como a hipertensão arterial, sendo esta uma doença prevalente em pacientes obesos do que em não obesos (Melo, 2011), (Camilo et al., 2010).

Hipertensão arterial (HA) é caracterizada como uma doença crônica multifatorial de grande frequência na população brasileira e mundial, no qual, há uma elevação sustentada dos 
níveis pressóricos $\geq 140$ e/ou $90 \mathrm{mmHg}$. Na maioria das vezes estão associadas a distúrbios metabólicos, alterações funcionais e/ou estruturais de órgãos-alvo, podendo ser agravada pela presença de outros fatores de risco, como dislipidemia, diabetes melito (DM), intolerância à glicose e obesidade abdominal (CARDIOL,2016). De acordo com a Sociedade Brasileira de Hipertensão (SBH 2017) até 2015 em países em desenvolvimento como o Brasil, o número de hipertensos poderá crescer $80 \%$.

Este trabalho tem como objetivo avaliar a correlação entre obesidade e hipertensão arterial em adultos jovens sedentários na cidade de Vitória da Conquista- Bahia.

\section{Metodologia}

Trata-se de um estudo com delineamento epidemiológico transversal, de cunho retrospectivo e descritivo e abordagem quantitativa, realizado no município de Vitória da Conquista, Bahia. Esta cidade possui aproximadamente 306.866 habitantes e está localizado há $509 \mathrm{~km}$ de distância da capital Salvador, apresenta coordenada geográfica como latitude $-14^{\circ}$ 53' e longitude - 40 48' (IBGE, 2010).

Os dados foram coletados em 2017, utilizando o método de abordagem aleatória estratificada. A população foi constituída por 178 indivíduos adultos de faixa etária estabelecida, com idade entre 22 a 45 anos. . A análise foi realizada com o auxílio do programa estatístico SPSS $® 24.0$, mediante aplicação de análise estatística descritiva e dos testes de Quiquadrado e Exato de Fisher. Para a coleta de dados foram realizadas entrevistas com os pacientes utilizando um instrumento de pesquisa sobre a forma de questionário que continham as variáveis demográficas (sexo e escolaridade); variáveis antropométricas.

De acordo Marconi; Lakatos (2010), este tipo de instrumento semiestruturado "permite explorar mais amplamente uma questão". Para aqueles que concordaram em participar do projeto de pesquisa será solicitada a assinatura do Termo de Consentimento Livre e Esclarecido em duas vias, uma para o pesquisador e a outra entregue ao paciente. A coleta de dados ocorreu em local privado e reservado.

Para obter os indicadores antropométricos foi utilizada uma balança portátil digital eletrônica, com capacidade de $150 \mathrm{Kg}$ (marca: Omron HBF 514C) para obter o peso e IMC. A estatura foi aferida utilizando estadiômetro portátil (marca: WCS), com plataforma anexa, com extensão de 2 metros e precisão de $1 \mathrm{~mm}$. A medição das circunferências da cintura foi realizada 
com auxílio de uma fita métrica flexível e inelástica com extensão de $2 \mathrm{~m}$, dividida em cm e subdividida em mm, por um único examinador. A medição da circunferência abdominal (CA) foi realizada 2,5 cm acima do umbigo, ao final da expiração. Os pontos de cortes estabelecidos segundo a OMS, (2000) para mulheres são de $\geq 85 \mathrm{~cm}$ e para homens $\geq 95 \mathrm{~cm}$.

A pesquisa foi aprovada pelo Comitê de Ética em Pesquisa da Faculdade Independente do Nordeste- FAINOR, e convergiu com os preceitos éticos dispostos na Resolução no 466/12 do Conselho Nacional de Saúde (CNS).

\section{Resultados}

Dos 178 pacientes, $68,5 \%$ são do sexo feminino e 31,5\% do sexo masculino. Esses dados apontam que, no grupo pesquisado, há um número maior de indivíduos do gênero feminino, sendo assim, mais prevalentes. A distribuição dos dados em frequência absoluta das variáveis analisadas neste estudo pode ser vista na tabela 1 .

Tabela 1 - Descrição dos dados obtidos dos sujeitos de pesquisa sobre circunferência abdominal versos hipertensão.

\begin{tabular}{|l|r|c|c|c|}
\hline \multirow{4}{*}{ Circunferência abdominal X Hipertensão } \\
\hline \multirow{3}{*}{ Feminino } & Hipertensão & Faixa Ideal & $\begin{array}{c}\text { Risco } \\
\text { Aumentado }\end{array}$ & Total \\
\cline { 2 - 5 } & Não & 3 & 4 & 7 \\
\cline { 2 - 5 } & Sim & 27 & 88 & 115 \\
\hline \hline \multirow{3}{*}{ Masculino } & Total & 30 & 92 & 122 \\
\cline { 2 - 5 } & Não & 2 & 0 & 2 \\
\cline { 2 - 5 } & Sim & 29 & 25 & 54 \\
\hline \hline \multirow{3}{*}{ Total } & Total & 31 & 25 & 56 \\
\cline { 2 - 5 } & Não & 5 & 4 & 9 \\
\cline { 2 - 5 } & Sim & 56 & 113 & 169 \\
\hline
\end{tabular}

Fonte: Pesquisa própria 2017.

Foram avaliados 178 indivíduos com idade entre 22 a 45 anos de ambos os sexos, no qual, foi avaliado a faixa ideal e o risco aumentado com relação à circunferência abdominal versus hipertensão, uma vez que, dos 178 pacientes, $65,7 \%$ se encontram com o risco 
aumentado e 34,3\% na faixa ideal de circunferência abdominal. Com relação à hipertensão 94,9\% dos pacientes são hipertensão e apenas 5,1\% não são hipertensos.

De acordo com os dados obtidos verificou-se que, das 122 mulheres avaliadas, 24,6\% estão na faixa ideal de circunferência abdominal e dentro desta faixa 90,0\% são hipertensas e apenas 10,0\% não apresentam hipertensão arterial, enquanto 75,4\% delas apresentam risco aumentado, sendo que $95,6 \%$ desta faixa são hipertensas e somente 4,4\% não são.

Com relação aos homens verificou-se que, dos 56 homens avaliados, 55,4\% estão na faixa ideal de circunferência abdominal e dentro desta faixa 96,7\% são hipertensos e apenas $3,3 \%$ não apresentam hipertensão arterial, enquanto os outros 44,6\% deles apresentam risco aumentado, sendo que, $100 \%$ são hipertensos.

\section{Discussão}

Os resultados deste estudo foram obtidos a partir de uma amostra de 178 adultos jovens, de ambos os sexos, na cidade de Vitória da Conquista, Bahia. Com a análise dos dados exibidos nos resultados foi possível conhecer característica antropométrica e com isso, identificar a correlação da obesidade com a hipertensão arterial. Foi analisado o indicador antropométrico tradicional, circunferência abdominal correlacionado com a hipertensão arterial.

Segundo Rezende e colaboradores (2006), um estudo realizado na Divisão de Saúde da Universidade Federal de Viçosa (UFV) diz que, a circunferência abdominal em mulheres apresenta mais elevada do que em homens o que corroboram com os achados no presente estudo, visto que, $75,4 \%$ das mulheres se encontram no risco aumentado. Evidentemente, há uma predominância de obesidade abdominal em mulheres podendo ser atribuída a maior concentração de gordura na região abdominal devido a diferença de hormônios, a gestações, como mostra Pinho e colaboradores (2013).

Com base nos estudos de Burgos e colaboradores (2013) realizado no município de Santa Cruz do Sul (RS) com crianças e adolescentes, a avaliação da circunferência abdominal (CA) se aplica como um bom marcador para detectar as alterações da pressão arterial, trazendo esse dado em comparativo com a pesquisa, que, apesar de apresentar um público alvo diferente, adultos jovens, o marcador antropométrico circunferência abdominal se mostrou eficaz quando associado à hipertensão arterial. 
A Sociedade Brasileira de Hipertensão (SBH) afirma que, o aumento do peso pode ser considerado um fator de risco para o desenvolvimento da hipertensão arterial, sendo responsável por $20 \%$ a $30 \%$ dos casos e que a perda de peso e a redução da circunferência abdominal podem contribuir para a redução da pressão arterial. No presente estudo, a prevalência de hipertensão arterial ficou em torno de 63,5\% para os pacientes que se encontram no risco aumentado de circunferência abdominal.

Estudos realizados Conde e Borges (2011) no Departamento de Nutrição da Faculdade de Saúde Pública da Universidade de São Paulo (USP) com adultos brasileiros mostra que, a ocorrência de obesidade é superior entre os homens, principalmente entre aqueles que são préobesos aos 20 anos e essa persistência do quadro de obesidade se torna frequente ao longo do tempo, dados esses que contradizem com os encontrados na nossa pesquisa, em virtude disso, acreditamos que o fato se deve por ele ter estudados indivíduos adultos de faixa etária diferente.

No presente estudo a prevalência de hipertensão arterial foi maior nos pacientes do sexo masculino em relação ao sexo feminino o que corroboram com os dados da pesquisa de Silva e colaboradores (2016) sendo $26,9 \%$ para o sexo masculino e $17,6 \%$ para o sexo feminino.

\section{Considerações Finais}

As doenças não transmissíveis atingiram um estágio preocupante para a saúde publica, com isso, a obesidade e a hipertensão que há poucas décadas acometiam uma população restrita de adultos, ganhou uma grande proporção chegando a todas as faixas etárias de forma semelhante.

A presente pesquisa evidenciou que, existe um aumento do sobrepeso e da obesidade na população brasileira, sendo muitas vezes associada com fatores de risco cardiovasculares, no qual, foi reforçada em nosso estudo. Com isso, pode-se utilizar intervenções que visam à redução do peso corporal, principalmente à gordura central, que é de extrema importância para a prevenção e controle das doenças cardiovasculares na população.

Desta forma, como dos 178 pacientes avaliados encontrou-se hipertensos tanto na faixa ideal de circunferência abdominal quanto na faixa de risco aumentado, faz se necessário à realização de outros exames físicos, investigação clínica e laboratorial como forma de confirmação de diagnostico. 


\section{Referências}

Associação Brasileira para Estudo da Obesidade e Síndrome Metabólica - ABESO. Diretrizes brasileiras de obesidade, 4.ed, São Paulo, SP., 2016, p. 1-188

ANDRADE, I. S., SOUSA, B. R., MOITINHO, B. G., MACÊDO, I. O., ANDRADE, A. G. F., FACCHINETTI, J. B., \& DUARTE, S. F. P. Associação entre a Percepção da Imagem Corporal com Indicadores Antropométricos em Adolescentes. Id on Line Rev. Psic., v. 11, n. 35, p. 531$541,2017$.

BRASIL, Ministério da Saúde. Mais da metade dos adultos está acima do peso . 2015. Disponível em: <http://www.brasil.gov.br/saude/2015/08/mais-da-metade-dos-adultos-estaoacima-do-peso>. Acesso em: 16 set. 2017.

BURGOS, M. S., REUTER, C. P., BUERGOS, L. T., POHL, H. H., PAULI, L. T. S., HORTA, J. A., ... CAMARGO, M. Comparison analysis of blood pressure, obesity, and cardiorespiratory fitness in schoolchildren. Arquivos Brasileiros de Cardiologia, v. 94, n. 6, p.788793, 2010.

CONDE, W. L.; BORGES, C. O risco de incidência e persistência da obesidade entre adultos brasileiros segundo seu estado nutricional ao final da adolescência. Revista brasileira de epidemiologia, v. 14, Suppl 1, n. 1, p. 71-9, 2011.

GHARAKHANLOU, R., FARZAD, B., AGHA-ALINEJAD, H., STEFFEN, L. M., \& BAYATI, M. Anthropometric measures as predictors of cardiovascular disease risk factors in the urban population of Iran. Arquivos Brasileiros De Cardiologia, v. 98, p. 126-135, 2012

INSTITUTO BRASILEIRO DE GEOGRAFIA E ESTATÍSTICA - IBGE. Estimativa dos municípios 2016. Disponível em:< https://www.ibge.gov.br/> acesso em 16 setembro. 2017.

INSTITUTO BRASILEIRO DE GEOGRAFIA E ESTATÍSTICA - IBGE. Sinopse do Censo Demográfico 2010. Disponível em:< https://censo2010.ibge.gov.br/> acesso em 19 setembro. 2017.

MELO, M. E. De. Doenças Desencadeadas ou Agravadas pela Obesidade. 2011. Disponível em http://www.abeso.org.br/pdf/Artigo - Obesidade e Doencas associadas maio 2011.pdf. Acesso em: 10.10.2016.

MARIATH, A. B. et al. Obesidade e fatores de risco para o desenvolvimento de doenças crônicas não transmissíveis entre usuários de unidade de alimentação e nutrição. Cadernos de Saúde Pública, v. 23, n. 4, p. 897-905, 2007.

MALACHIAS, M., SOUZA, W., PLAVNIK, F., RODRIGUES, C., BRANDÃO, A., NEVES, M., MORENO JÚNIOR, H. VII Diretriz Brasileira De Hipertensão Arterial. Arquivos Brasileiros de Cardiologia, v. 107, n. 3, p. 1-83, 2016

ORGANIZAÇÃO MUNDIAL DA SAÚDE - OMS. Valores de referência antropometria. Disponível em:< http://www.paho.org/bra/> acesso em 20 setembro. 2017. 
PINHO, C. P. S., DINIZ, A. da S., ARRUDA, I. K. G. de, FILHO, M. B., COELHO, P. C., SEQUEIRA, L. A. de S., \& LIRA, P. I. C. de. Prevalência de excesso de peso e obesidade e fatores associados, Brasil, 2006. Cad. Saúde Pública, v. 43, n. 2, p. 83-89, 2013

REZENDE, F. A. C., ROSADO, L. E. F. P. L., RIBEIRO, R. de C. L., VIDIGAL, F. de C., VASQUES, A. C. J., BONARD, I. S., \& CARVALHO, C. R.. Body mass index and waist circumference: association with cardiovascular risk factors. Arquivos Brasileiros de Cardiologia, v. 87, n. 6, p. 728-734, 2006

SOUZA, I. F. DA S. et al. Elderly with knee osteoarthritis should perform nutritional assessment: integrative literature review. Einstein, São Paulo, v. 15, n. 2, p. 226-232, 2017.

FERNÁNDES C. DANIELLA et al. Obesity and asthma: association or coincidence? J Pediatria, Rio de Janeiro, v. 86, n. 1, p. 6-14, 2010.

\section{Como citar este artigo (Formato ABNT):}

ORMUNDO, Larissa S.; DUARTE, Stênio F. P. Avaliar a Correlação da Obesidade com Hipertensão Arterial em Adultos Sedentários na Cidade de Vitória da Conquista. Id on Line Revista Multidisciplinar e de Psicologia, 2017, vol.11, n.38, p.791-798. ISSN: 1981-1179.

Recebido: 08.11.2017

Aceito: 10.11 .2017 